\title{
Commonly used caspase inhibitors designed based on substrate specificity profiles lack selectivity
}

\author{
Alicia B Berger ${ }^{1,2}$, Kelly B Sexton ${ }^{1}$, Matthew Bogyo ${ }^{1,3}$ \\ ${ }^{1}$ Department of Pathology, Stanford University School of Medicine, 300 Pasteur Dr., Stanford, CA 94305, USA; ${ }^{2}$ Cancer Biology \\ Graduate Program, Stanford University School of Medicine, 300 Pasteur Dr., Stanford, CA 94305, USA; ${ }^{3}$ Microbiology and Immu- \\ nology, Stanford University School of Medicine, 300 Pasteur Dr., Stanford, CA 94305, USA
}

Cell Research (2006) 16:961-963. doi: 10.1038/sj.cr.7310112; published online 21 November 2006

\section{Dear Editor:}

Caspase regulation and activation have been extensively studied since the discovery of this class of proteases almost two decades ago, yet surprisingly few tools are available that can be used to monitor individual caspase activities [1]. The most commonly used tools include caspase-specific anti-sera as well as fluorogenic substrates and inhibitors. Unfortunately, antibody reagents often do not provide an accurate measure of caspase activity since several caspase family members (caspases 8/10 and 9) do not require proteolytic processing for activation $[2,3]$. Furthermore, recent evidence suggests that caspase-7 (an executioner caspase) activation occurs via a catalytically active full-length intermediate that cannot be differentiated from the non-cleaved inactive zymogen using antibodies [4, 5].

As an alternative to antibody-based reagents, small molecule substrates and inhibitors can be used to directly monitor caspase activity and a relatively large number of caspase substrates, inhibitors, and activity-based probes are now commercially available. These reagents have begun to find widespread use in cell biological and biochemical studies of caspase function. In most cases, these tools are used as selective reagents to assess the contribution of specific caspases to a given apoptotic pathway.

Virtually, all of the current commercially available reagents have been designed based on the reported optimal substrate specificity profiles for the caspases [6]. However, these primary peptide sequences may provide only limited selectivity when used in the context of a small molecule

Correspondence: Matthew Bogyo

Tel: +1-650-725-4132; Fax: +1-650-725-7424;

E-mail: mbogyo@stanford.edu inhibitor. Interestingly, a comprehensive study of the selectivity of the most commonly used commercially available caspase inhibitors has never been reported. Thus, we felt that a study of the selectivity of these inhibitors towards endogenous caspases in complex proteomes would be extremely valuable. We chose to focus on three widely used covalent caspase inhibitors containing the optimal substrate specificity sequences DEVD, I/LETD, and LEHD designed to target caspases $3 / 7,8$, and 9 , respectively. For these studies, we obtained three commercially available inhibitors (Z-DEVD-FMK, Z-IETD-FMK, and Z-LEHDFMK) equipped with a fluoromethylketone (FMK) reactive group. We also synthesized three inhibitors that contained the same peptide sequences (NP-DEVD-AOMK, NPLETD-AOMK, and NP-LEHD-AOMK) but linked to the acyloxymethylketone (AOMK) reactive group shown previously to be effective towards caspases [7, 8] (Figure 1A). To test inhibitor selectivity against caspases 3,7 , and 9 we used an apoptotic proteome in which the intrinsic apoptotic pathway is activated through addition of cytochrome $c$ and dATP [9]. Since there is currently no simple method to monitor endogenous caspase- 8 activity, we tested the inhibitors against purified recombinant caspase- 8 at the physiologically relevant concentration of $50 \mathrm{nM}$ (See Supplement 1 for Materials and Methods) [2].

To assess caspase inhibition, we added cytochrome $c /$ dATP to cytosolic 293 extract and allowed the samples to incubate for $10 \mathrm{~min}$ at $37^{\circ} \mathrm{C}$ at which time the panel of inhibitors was added at a range of concentrations and allowed to inhibit active caspases for $10 \mathrm{~min}$. After incubation, the poly-caspase probe KMB01 (biotin-hex-EVD-AOMK) [8] was added to label residual caspase active sites for an additional $30 \mathrm{~min}$. After 10-min incubation with cytochrome $c$ and dATP, KMB01 labels the fully cleaved forms of 20 
A

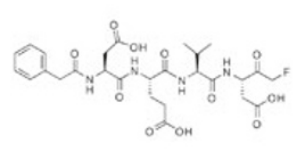

Z-DEVD-FMK

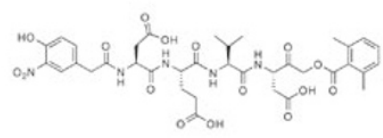

NP-DEVD-AOMK

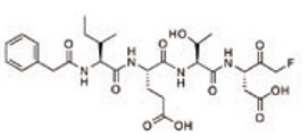

Z-IETD-FMK

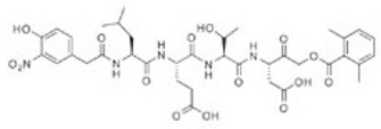

NP-LETD-AOMK

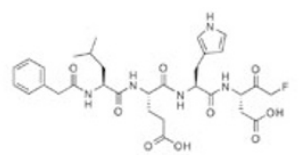

Z-LEHD-FMK

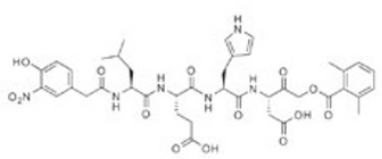

NP-LEHD-AOMK

B

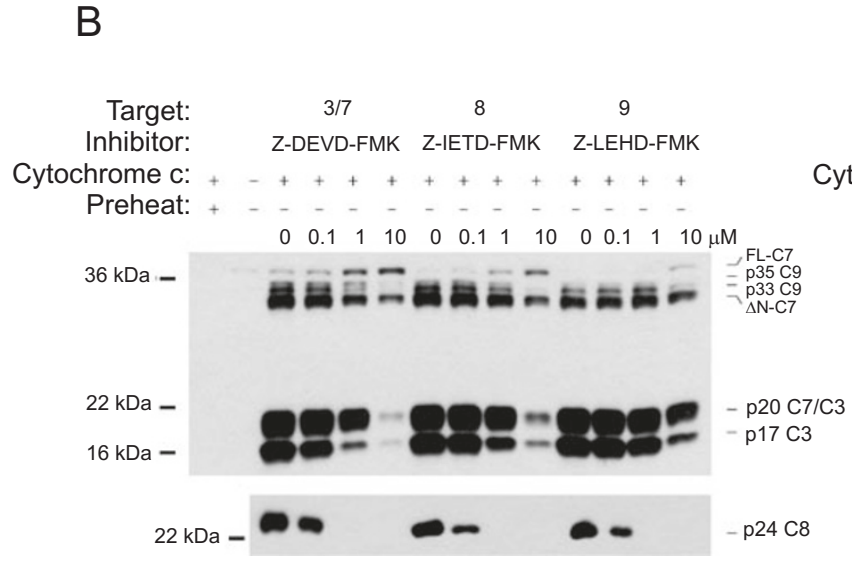

C

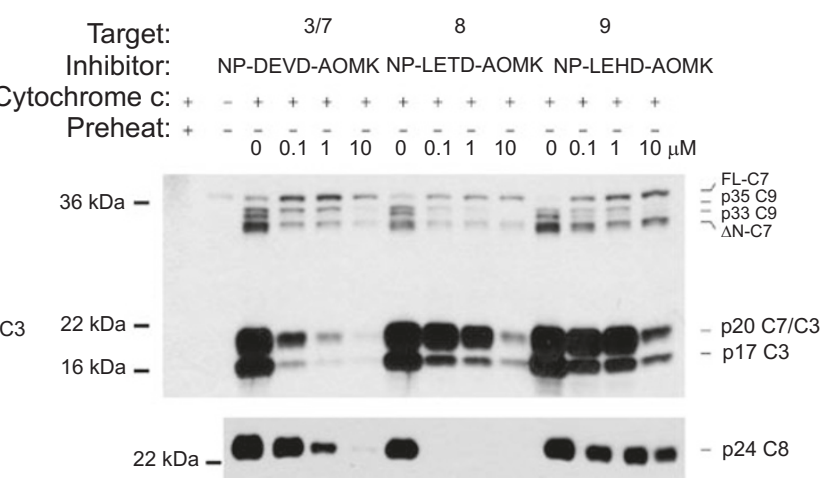

Figure 1 (A) Structures of FMK and AOMK inhibitors. Indirect competition of FMK (B) and AOMK (C) inhibitors with active caspases 3,7 , and 9 in apoptotic cytosolic extract (top panels) and with active recombinant caspase-8 (lower panels; note: cytochrome $c /$ dATP were not added to caspase-8 samples). Residual caspase active sites were labeled with $20 \mu \mathrm{M}$ KMB01 (Biotin-hex-EVD-AOMK) for $30 \mathrm{~min}$ after inhibitor treatment at the concentrations indicated. Samples were analyzed by SDS-PAGE followed by biotin blotting using streptavidin-HRP (Sigma). Caspases are represented as follows: FL-C7, p35 C9 (35 kDa form of caspase-9), p33 C9 (33 kDa form of caspase-9), p20 C7/C3 (20 kDa forms of caspases 3 and 7), p17 C3 (17 kDa form of caspase-3), and p24 C8 (24 kDa form of caspase-8). FL-C7 accumulates over time in cytosolic extracts due to inhibition of downstream executioner caspases consistent with the hypothesis that processing of active FL-C7 to fully processed mature forms is carried out by executioner caspases [5].

$\mathrm{kDa}$ forms of caspases 3 and 7 and the $17 \mathrm{kDa}$ form of caspase-3. A 37-kDa active full-length form of caspase7 (FL-C7) and a 33-kDa active form of caspase-7 with an $\mathrm{N}$-terminal cleavage $(\triangle \mathrm{N}-\mathrm{C} 7)$ are also visible. Active caspase-9 is present as 33 and $35 \mathrm{kDa}$ active forms. The identification of all caspases has been confirmed through immunoprecipitation experiments using specific anti-sera [5]. Recombinant caspase- 8 was pre-activated in caspase buffer (100 mM Tris, $2 \mathrm{mM}$ DTT, 0.1\% CHAPS, 10\% sucrose, $\mathrm{pH}$ 7.4) for $10 \mathrm{~min}$ followed by a 30-min incubation with inhibitor and a 30-min incubation with KMB01 (Figure $1 \mathrm{~B}$ and $1 \mathrm{C}$ ). Active caspase- 8 is visible as a single $24 \mathrm{kDa}$ form in this assay.

The results of our study clearly show that all inhibitors tested demonstrate significant cross-reactivity towards nontarget caspases. In general, the AOMK warhead slightly increased selectivity and increased potency towards the desired target caspase relative to the FMK reactive group. In particular, the caspase- 3 targeted inhibitors Z-DEVD-FMK and NP-DEVD-AOMK completely inhibited caspase-9 at high concentrations and showed significant cross-reactivity towards caspase- 8 at all concentrations tested. The caspase- 8 targeted compounds Z-IETD-FMK and NP-LETD-AOMK did show good potency towards caspase-8, and also showed significant cross-reactivity towards caspases 3 and 9 . The two caspase-9 targeted compounds (Z-LEHD-FMK and NPLEHD-AOMK) were weak caspase-9 inhibitors yet showed relatively good potency towards caspases 3 and 8 suggesting that the LEHD, in the context of an irreversible inhibitor, is a particularly poor selectivity sequence for caspase- 9 . The results of this study and others [5] suggest that crossreactivity with endogenous caspase- 3 may be unavoidable due to the high concentrations of active caspase- 3 relative to other caspases. However, due to their lower endogenous 
concentrations, selectivity between caspases 8 and 9 may be achieved with better inhibitor design. Achieving selectivity between caspases 8 and 9 is especially important for experiments designed to distinguish between the contributions of intrinsic and extrinsic apoptosis pathways.

The overall purpose of this communication is to highlight the caveats of using small molecule caspase inhibitors to assess the role of specific proteases in a given biological process. In particular, extreme care should be taken when using covalent inhibitors that contain peptide sequences based only on optimal substrate profiles. In our small study of covalent inhibitors, we have shown that none of the compounds are highly selective and all exhibit broad inhibition when used at high concentrations. Furthermore, it is possible that other "selective" caspase inhibitors designed to target other caspases may demonstrate similar cross-reactivity when used at concentrations required for cell-based studies. One must be aware of this lack of selectivity both when carrying out experiments and when evaluating studies in which claims are made regarding the role of individual caspases based solely on "selective" inhibitor studies.

As an increasing number of selective reagents become available some of the issues of cross-reactivity that we currently face may become less of an obstacle; however, comprehensive studies using multiple reagents and methods will be required to confirm the selectivity of any new reagents and the selectivity of commercially marketed compound should be verified by the end user. Only in the case where target selectivity can be verified in relevant biological samples should small molecule inhibitors be used to make claims of specific caspase function.

\section{Acknowledgments}

This work was supported by an NIH National Technology Center for Networks and Pathways Grant U54 RR020843 (to MB) and the NIH Grant R01-EB005011 (to MB). AB was funded by an NHGRI training Grant 5T32 HG00044.

\section{References}

1 Janicke RU, Sohn D, Totzke G, Schulze-Osthoff K. Caspase-10 in mouse or not? Science 2006; 312:1874.

2 Boatright KM, Renatus M, Scott FL, et al. A unified model for apical caspase activation. Mol Cell 2003; 11:529-541.

3 Pop C, Timmer J, Sperandio S, Salvesen GS. The apoptosome activates caspase-9 by dimerization. Mol Cell 2006; 22:269-275.

4 Denault JB, Bekes M, Scott FL, Sexton KM, Bogyo M, Salvesen GS. Engineered hybrid dimers: tracking the activation pathway of caspase-7. Mol Cell 2006; 23:523-533.

5 Berger $\mathrm{AB}$, Witte $\mathrm{MD}$, Denault JB, et al. Identification of early intermediates of caspase activation using selective inhibitors and activity-based probes. Mol Cell 2006; 23:509-521.

6 Thornberry NA, Rano TA, Peterson EP, et al. A combinatorial approach defines specificities of members of the caspase family and granzyme B. Functional relationships established for key mediators of apoptosis. J Biol Chem 1997; 272:17907-17911.

7 Thornberry NA, Peterson EP, Zhao JJ, Howard AD, Griffin PR, Chapman KT. Inactivation of interleukin-1 beta converting enzyme by peptide (acyloxy)methyl ketones. Biochemistry 1994; 33:3934-3940.

8 Kato D, Boatright KM, Berger AB, et al. Activity-based probes that target diverse cysteine protease families. Nat Chem Biol $2005 ; \mathbf{1}: 33-38$.

9 Liu X, Kim CN, Yang J, Jemmerson R, Wang X. Induction of apoptotic program in cell-free extracts: requirement for dATP and cytochrome $c$. Cell 1996; 86:147-157. 